\title{
Article
}

Mycosphere

\section{Exploration of wild medicinal mushroom species in Walayar valley, the Southern Western Ghats of Coimbatore District Tamil Nadu.}

\author{
Venkatachalapathi $\mathbf{A}^{\mathbf{1}}$ and Paulsamy $\mathbf{S}^{\mathbf{1}}$ \\ ${ }^{1} P G$ and Research Department of Botany, Kongunadu Arts and Science College, Coimbatore - 641029.
}

Venkatachalapathi A, Paulsamy S 2016 - Exploration of wild medicinal mushroom species in Walayar valley, the Southern Western Ghats of Coimbatore District Tamil Nadu. Mycosphere 7(2), 118-130, Doi 10.5943/mycosphere/7/2/3

\begin{abstract}
The present study explored the medicinal mushroom species used by the Irula tribal community in Walayar Valley, the southern Western Ghats of Coimbatore District, Tamil Nadu, India. The study was between May 2013 to August 2014 and reportson30medicinal mushroom species belonging to 23 genera in 13 families. The fungi occurred between 421 to $834 \mathrm{~m}$ above m.s.l. Mushrooms were recorded in separate field data sheets, including binomial name, local name, medicinal uses and quantitative assessment of mushroom species collected per season per $\mathrm{Kg}$ based on information provided by the Irula community. These species included treatment of eight different illness categories. The study concludes their nutritional and medicinal potential, as well as ethno medicinal uses, which may have future pharmaceutical application.
\end{abstract}

Key words - Irula - medicinal mushroom - Walayar valley - Western Ghats

\section{Introduction}

Nature is a source of therapeutic agents and an impressive number of modern drugs have been isolated from natural resources, many of the drug isolations were based on traditional medicinal uses (Kinsalin et al. 2014). Mushrooms are spore-bearing, fleshy fruiting bodies of the fungi, that are typically produced above ground soil (Chandulal et al. 2013, Sharma \& Atri 2014, Stadler \& Hoffmeister 2015). They are widely consumed and are an edible and medical resource (Mortimer et al. 2012, Hilden et al. 2013, Thawthong et al. 2014, Bandara et al. 2015). Many mushrooms have therapeutic properties, such as antioxidant, antimicrobial, anticancer, cholesterol lowering and immuno stimulatory effects (Barros et al. 2007, Oyetayo 2009, Villares et al. 2012, Da Silva et al. 2012, De Silva 2012a, b, 2013, Lin et al. 2013, Liu 2014, Bandara et al. 2015, Benjarong et al. 2015) as they contain a variety of secondary metabolites, including phenolic compounds, polypeptides, triterpenes and steroids (Ziegenbein et al. 2006, Turkoglu et al. 2007, Dai et al. 2009, Liu et al. 2011, Luo et al. 2013, Thatoi \& Singdevsachan 2014). Many genera of mushrooms are edible and are rich in essential nutrients, such as carbohydrates, and have a low fat and oil content, and contain proteins, vitamins, mineral, fibres and various amino acids (Fasidi \& Kadiri 1990, Ziegenbein et al. 2006, Hyde et al. 2010, Rios et al. 2012, Jing Xu et al. 2014, Luangharn et al. 2014, Bandara et al. 2015). 

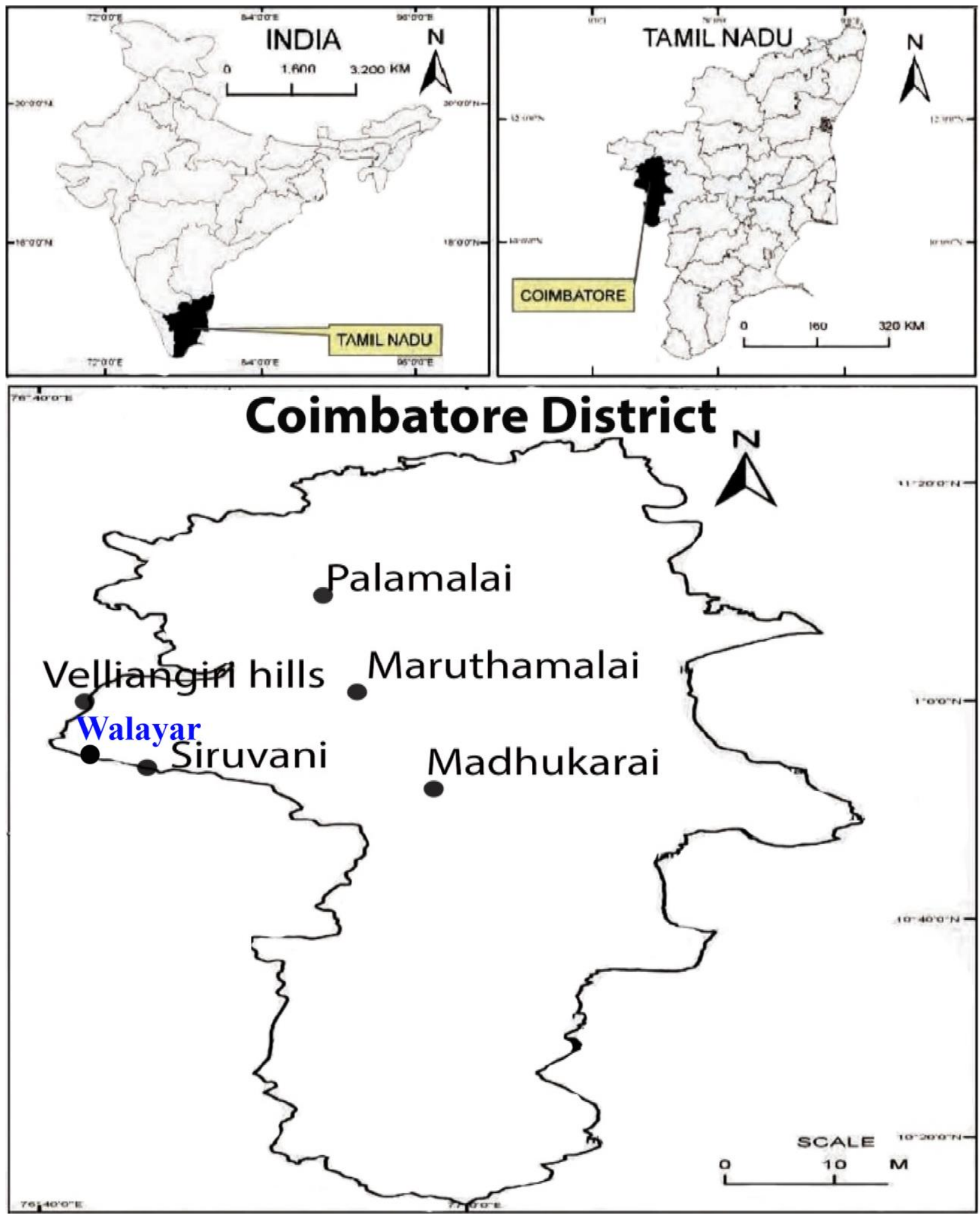

Figs 1 - Map showing the location of Walayar valley.

Walayar Valley, which is an important landscape situated in Tamil Nadu and Kerala parts of Western Ghats, harbours a rich variety of plants and edible mushrooms. The tribal community in this region, the Irula, use several edible mushrooms for their day-today life. The present study lists the edible mushroom species used by this group based on information provided by the indigenous tribal community. 


\section{Materials \& Methods}

\section{Study Area}

The geographical location of the Walayar Valley is $76^{\circ} 52^{\prime} 0^{\prime \prime}-76^{\circ} 52^{\prime} 21^{\prime \prime} \mathrm{E}$ and $10^{\circ} 23^{\prime} 30^{\prime \prime}-$ $10^{\circ} 23^{\prime} 52^{\prime}$ 'N. It spreads in an area of ca.1100 ha both in Tamil Nadu and Kerala parts of Western Ghats (Fig. 1). The altitude of the valley lies between 410 and $892 \mathrm{~m}$ above m.s.l. The annual rainfall ranges between 2300 to $3200 \mathrm{~mm}$ for the past fifteen years. The vegetations are mostly moist deciduous and semi-evergreen forests (Champion \& Seth 1968). The study was made in Tamil Nadu part of Western Ghats (ca. 550 ha).

\section{Collection and identification of mushroom species}

The mushroom species were collected in Walayar Valley during the period from May 2013 to August 2014. For their identification, taxonomic keys and descriptions were consulted (Gillman 1957). Descriptions of basidiomycetes were made according to their macro, micro and cultural features by using standard manual, The Manual of Soil Fungi (Ellis 1971).

\section{Ailment categories}

Eight ailment categories were grouped (Cook 1995) on basis of the information provided by the Irula healers of Walayar Valley (Table 1). They are gastro-intestinal ailments (GIA), dermatological infections/diseases (DID), respiratory system diseases (RSD), genito-urinary ailments (GUA), skeleto-muscular system disorders (SMSD), circulatory system/cardiovascular diseases (CSCD), endocrinal disorders (ED) and liver problems (LP).

\section{Results\& Discussion}

Several naturally growing wild, medicinal mushrooms could be found in Walayar Valley. A total number of 30 mushroom species, belongs to 23 genera in 15 families was collected (Fig. 2). The detailed information about the botanical and common names of the mushroom species, family and habitat, medicinal uses with their reference, are presented in Table 2. Based on species contribution, the families viz., Agaricaceae (5 species), Pleurotaceae (4 species), Polyporaceae (4 species), Tricholomataceae (3 species) and Lyophyllaceae (3 species) were dominant. The other families like Sclerodermataceae (2 species), Russulaceae (1 species), Hygrophoraceae (1 species), Ganodermataceae (1 species), Coriolaceae (1 species), Clavulinaceae (1 species), Lycoperdaceae (1 species), Pluteaceae (1 species), Auriculariaceae (1 species) and Marasmiaceae (1 species) were also present with less than 1 or 2 species. All these mushroom species are used as by the Irula tribal community in that region, whenever they are available. This wide diversity of mushroom species may be due to the existence of different types of vegetations and several microclimatic sites. Pushpa \& Purushothama (2012) found that the presence of varied types of major communities naturally offer specific localities for taxonomically differing macrofungi.

The 30 species of medicinal mushroom fungi used by the Irula tribal community, that are classified as 8 different ailment categories (Cook 1995), and are already reported as edible elsewhere (Rajesh et al. 2013, Tapwal et al. 2013, Mohanan 2014) and no new report was made for these species in the present study. The tribal healers further informed that the medicinal mushroom species are collected at different months at various altitudes ranging between the 421 to $834 \mathrm{~m}$ above m.s.l. and the quantitative of mushroom species collected per season/Kg are clearly recorded in this region (Table 3). This includes Auricularia auricula, Agaricus augustus, A. bisporus, A. campestris, A. heterocystis, Bovista nigrescens, Clavulina rugosa, Clitocybe nuda, Coprinus sp., Ganoderma lucidum, Hygrocybe sp., Lentinus sajor-caju, Marasmius androsaceus, Melanoleuca grammopodia, Mycena galericulata, Pisolithus arhizus, Pleurotus ostreatus, P. sajor-caju, P. Sapidus, Russula fragilis, Scleroderma citrinum, Termitomyces heimii, T. microcarpus, Trametes versicolor and Volvariella speciosa). 
Table 1 Irulas ailments grouped by illness category (Cook 1995).

\begin{tabular}{|c|c|c|c|}
\hline S. No. & Ailment categories & Biomedical terms & Tamil terms \\
\hline 1. & $\begin{array}{l}\text { Circulatory system/ } \\
\text { cardiovascular diseases (CSCD) }\end{array}$ & $\begin{array}{l}\text { Cardiac disorders } \\
\text { Blood purification } \\
\text { Blood pressure/ hypertension } \\
\text { Arteries problem } \\
\text { Improve immune system }\end{array}$ & $\begin{array}{l}\text { Idhaya noi } \\
\text { Rattha sutthigarippu } \\
\text { Rattha alutham } \\
\text { Thamani thontharavu } \\
\text { Noi ethirpu sakthi athigaripu }\end{array}$ \\
\hline 2. & $\begin{array}{l}\text { Dermatological } \\
\text { infections/diseases (DID) }\end{array}$ & $\begin{array}{l}\text { Cuts } \\
\text { Wound healing } \\
\text { Skin diseases } \\
\text { Sores }\end{array}$ & $\begin{array}{l}\text { Vettukkayam } \\
\text { Kaayam } \\
\text { Thol noi } \\
\text { Pungal }\end{array}$ \\
\hline 3. & Endocrinal disorders (ED) & $\begin{array}{l}\text { Diabetes } \\
\text { Enhance insulin secretion } \\
\text { Kidney problem } \\
\text { Urinary problem }\end{array}$ & $\begin{array}{l}\text { Sarkkarai/neerilivu noi } \\
\text { Kanaiya sueapu neer athigarika } \\
\text { Siruneeraga kal } \\
\text { Siruneeraga thontharavu }\end{array}$ \\
\hline 4. & Gastro-intestinal ailments (GIA) & $\begin{array}{l}\text { Ulcer } \\
\text { Digestion/indigestion } \\
\text { Dysentery } \\
\text { Cholesterol }\end{array}$ & $\begin{array}{l}\text { Vayitru pun } \\
\text { Geeranam/ageeranam } \\
\text { Seedhabaethi } \\
\text { Kollupu }\end{array}$ \\
\hline 5. & Genito-urinary ailments (GUA) & Piles & Mula noi \\
\hline 6. & Liver problem (LP) & Liver problem & Kalliral noiku tanik \\
\hline 7. & Respiratory system diseases (RSD) & Cough & Irumal \\
\hline 8. & $\begin{array}{l}\text { Skeleto-muscular } \\
\text { system disorders (SMSD) }\end{array}$ & $\begin{array}{l}\text { Rheumatism } \\
\text { Arthritis } \\
\text { Tumor } \\
\text { Swelling/inflammation/ } \\
\text { Nervous problem } \\
\text { Depression }\end{array}$ & $\begin{array}{l}\text { Moottu vadham } \\
\text { Kilvatam } \\
\text { Putru noi } \\
\text { Veekam } \\
\text { Narambu pathipu } \\
\text { Mana alutham }\end{array}$ \\
\hline
\end{tabular}

Medical mycology is the traditional use of mushrooms. They have been used in medicine since the Neolithic and Paleolithic eras (Samorini 2001). Although mushrooms have been used in China as medicine since 100 A.D. (Gunde 1999), it was only in 1960 that scientists investigated the basic active principles of mushrooms which are health promoting. Mushrooms have been used in health care for treating simple and age old common diseases, such as skin diseases and pandemic diseases such as Cardiac disorders, cancer/tumour and liver problems. Interestingly, it was found out from the Irula tribal healers that certain species viz., Ganoderma lucidum, Lentinus tuberegium, Lycoperdon echinatum, Lentinus squarrosulus, Termitomyces heimii, Auricularia auricula, Termitomyces microcarpus and Pleurotus sajor-caju are also used for the treatment of common ailments,such as fever, coughs and fungal infections.

Mushrooms are an important bioresource of novel secondary metabolites (Aletor 1995, Mattila et al. 2000, Deshmukh 2004, Kala 2009, Guillamon et al. 2010, Johnsy et al. 2011, Davidson et al. 2012, Sachan et al. 2013, Semwal et al. 2014, Qin et al. 2015). In India, alternative systems of medicine, utilize the curative properties of mushrooms. The secondary metabolites from these mushrooms are chemically diverse and possess a wide spectrum of biological activities (Rai et al. 2005). In India, several mushrooms have been reported as medicinal mushrooms, which have antioxidant, antimicrobial, anti-inflammatory activity with antitumor and other properties.

The ethnological aspects of mushrooms have also been reported by workers in different parts of India as well as worldwide (Harsh et al. 1993, Bulakh 2001, Bhosle et al. 2010, Bhaben et al. 2011, Davidson et al. 2012, Gogoi \& Sarma 2012, Khaund \& Joshi 2013, Kabita et al. 2014, Richter et al. 2015). Wild edible mushrooms have been reported from Manipur and Arunachal Pradesh of North East India (Sing \& Sing 1993, Sing et al. 2002) whereas, from Assam, Baruah et al. (1971) reported a few basidiomycetous fungus of Sibsagar District. In central India, Ganoderma lucidum is used as herbal medicine by the Baiga tribes to cure asthma, Agaricus sp. is used in goiter treatment and L. pusilum in wound healing and also for controlling bleeding (Rai et al. 2005). 
Table 2 Medicinal mushroom species used by the Irula tribal community for their ailments in Walayar Valley, the Western Ghats of Coimbatore district, Tamil Nadu.

\begin{tabular}{|c|c|c|c|c|c|c|}
\hline $\begin{array}{l}\text { S. } \\
\text { No. }\end{array}$ & Botanical Name & $\begin{array}{c}\text { Common } \\
\text { name }\end{array}$ & Family & Habitat of growth & Medicinal uses & Reference \\
\hline 1 & Auricularia auricular (Bull.) J. & Jew's ear & Auriculariaceae & $\begin{array}{l}\text { Upon wood of } \\
\text { deciduous } \\
\text { trees and shrubs }\end{array}$ & $\begin{array}{l}\text { Cardiovascular diseases, } \\
\text { diabetes and hypertension. }\end{array}$ & $\begin{array}{l}\text { Yuan et al. 1998, } \\
\text { Guillamon et al. 2010, } \\
\text { Kabita et al. } 2014 .\end{array}$ \\
\hline 2 & Agaricus augustus Fr. & Not known & Agaricaceae & Deciduous woods & $\begin{array}{l}\text { High cholesterol, arteries } \\
\text { problem and ulcer. }\end{array}$ & $\begin{array}{l}\text { Poongkode et al. } 2001 \text {, } \\
\text { Fortes et al. } 2009 .\end{array}$ \\
\hline 3 & A. bisporus (J.E.Lange) Emil J. & $\begin{array}{l}\text { White button } \\
\text { mushroom }\end{array}$ & Agaricaceae & Grassy places & Enhance insulin secretion. & $\begin{array}{l}\text { Ahmad 1984a, Jagadish et } \\
\text { al. } 2009 \text {. }\end{array}$ \\
\hline 4 & A. campestris $\mathrm{L}$. & $\begin{array}{l}\text { Meadow } \\
\text { mushroom }\end{array}$ & Agaricaceae & Sides of pathways & Diabetes and ulcer. & $\begin{array}{l}\text { Ewart et al. 1975, Ahmad } \\
\text { et al. 1984a, b, Gray \& } \\
\text { Flatt } 1998, \text { Sun } 2014 .\end{array}$ \\
\hline 5 & A. heterocystis Heinem. & Not known & Agaricaceae & Grassy places & $\begin{array}{l}\text { Antitumor, cholesterol and } \\
\text { kidney problem. }\end{array}$ & $\begin{array}{l}\text { Bankhead \&Charles 2000, } \\
\text { Ajith \& Janardhanan 2007, } \\
\text { Jagadish et al. 2009, } \\
\text { De Silva et al. 2012b. }\end{array}$ \\
\hline 6 & Bovista nigrescens Pers. & Brown puffball & Agaricaceae & Pastureland & $\begin{array}{l}\text { Broken skin or wound and stop } \\
\text { bleeding. }\end{array}$ & $\begin{array}{l}\text { Densmore 1928, Chang \& } \\
\text { Miles } 2004 .\end{array}$ \\
\hline 7 & Calocybe indica Kuhner ex Donk. & $\begin{array}{l}\text { Milky } \\
\text { mushroom }\end{array}$ & Lyophyllaceae & Evergreen forests & Diabetes & Chang 1996, Sun 2014. \\
\hline 8 & Clavulina rugosa (Fr.) Schroet. & $\begin{array}{l}\text { Wrinkled coral } \\
\text { fungus }\end{array}$ & Clavulinaceae & Rotten wood & Skin diseases & $\begin{array}{l}\text { Chang and Mao 1995, } \\
\text { Chang \& Miles } 2004 .\end{array}$ \\
\hline 9 & Clitocybe nuda (Bull.) H.E. & Wood blewit & Tricholomataceae & Decaying leaf litter & Cardiovascular. & $\begin{array}{l}\text { Guillamon et al.2010, } \\
\text { Kabita et al. } 2014 \text {. }\end{array}$ \\
\hline 10 & Coprinus sp. & Not known & Agaricaceae & $\begin{array}{l}\text { Buried wood or in } \\
\text { grass. }\end{array}$ & $\begin{array}{l}\text { Diabetes, circulatory disease, } \\
\text { digestive disorders and piles. }\end{array}$ & Wasser 2002, Sun 2014. \\
\hline 11 & Daedaleopsis confragosa (Bolton) J. & $\begin{array}{l}\text { Blushing } \\
\text { bracket }\end{array}$ & Polyporaceae & Tree trunk & Skin diseases & $\begin{array}{l}\text { Kasuga et al.1995, } \\
\text { Lindequist et al. } 2005 \text {. }\end{array}$ \\
\hline 12 & Ganoderma lucidum (Curtis) P. & Reishi & Ganodermataceae & $\begin{array}{l}\text { Stumps of } \\
\text { deciduous trees }\end{array}$ & $\begin{array}{l}\text { Improve immune system, liver } \\
\text { protection, lower blood } \\
\text { pressure and } \\
\text { inhibits cholesterol synthesis. }\end{array}$ & $\begin{array}{l}\text { Komoda et al. 1989,Lin } \\
\text { \&Zhang 2004, } \\
\text { Wang et al. 2007, } \\
\text { Moradali et al. 2007, } \\
\text { Sun \& Zhou 2014. }\end{array}$ \\
\hline 13 & Hygrocybe sp. (Schaeff.) P. & Not known & Hygrophoraceae & Tree trunks or logs. & Skin diseases & $\begin{array}{l}\text { Ohtsuka et al. 1997, } \\
\text { Chang \& Miles } 2004 .\end{array}$ \\
\hline 14 & Lentinus sajor-caju (Fries) Fries. & White-rot & Polyporaceae & Dead wood & Lower cholesterol and & Ajith \& Janardhanan 2007, \\
\hline
\end{tabular}




\begin{tabular}{|c|c|c|c|c|c|c|}
\hline $\begin{array}{l}\text { S. } \\
\text { No. }\end{array}$ & Botanical Name & $\begin{array}{c}\text { Common } \\
\text { name }\end{array}$ & Family & Habitat of growth & Medicinal uses & Reference \\
\hline & & Fungus & & & anti-cancer agent. & $\begin{array}{l}\text { Enman et al. } 2007, \\
\text { De Silva et al. } 2012 \mathrm{~b} .\end{array}$ \\
\hline 15 & L. squarrosulus Mont. & Not known & Polyporaceae & Cashew nut tree & Antiulcer. & Mattila et al. 2000. \\
\hline 16 & L. tuberregium (Fr.) Fr. & $\begin{array}{l}\text { White-rot } \\
\text { fungus }\end{array}$ & Polyporaceae & Dead wood & $\begin{array}{l}\text { Cough, indigestion and } \\
\text { dysentery. }\end{array}$ & Chang \& Lee 2004. \\
\hline 17 & Lycoperdon echinatum Pers. & Spiny puffball & Agaricaceae & $\begin{array}{l}\text { Deciduous forests } \\
\text { and grassy } \\
\text { areas or woody } \\
\text { debris }\end{array}$ & Wound healing & $\begin{array}{l}\text { Mitchell \& Perfect 1995; } \\
\text { Chang \& Miles } 2004 .\end{array}$ \\
\hline 18 & Marasmius androsaceus (L.) Fr. & Not known & Marasmiaceae & $\begin{array}{l}\text { Deciduous } \\
\text { hardwood trees }\end{array}$ & $\begin{array}{l}\text { Blood purification, } \\
\text { antiinflamatory, nerves } \\
\text { problem and rheumatism. }\end{array}$ & $\begin{array}{l}\text { Wasser \&Weis 1999a, Yu } \\
\text { et al. } 2009 .\end{array}$ \\
\hline 19 & Melanoleuca grammopodia Bull. & Not known & Tricholomataceae & Wood & Skin diseases & $\begin{array}{l}\text { Mohamed \& Dix } 1988, \\
\text { Chang \& Miles } 2004 .\end{array}$ \\
\hline 20 & Mycena galericulata (Scop.) Gray. & $\begin{array}{l}\text { Common } \\
\text { bonnet }\end{array}$ & Tricholomataceae & Submerged wood & Skin diseases & $\begin{array}{l}\text { Mohamed \& Dix 1988, } \\
\text { Chang \& Miles } 2004 .\end{array}$ \\
\hline 21 & Pisolithus arhizus (Scop.) Rauschert. & $\begin{array}{l}\text { Dye makers } \\
\text { puffball }\end{array}$ & Sclerodermataceae & $\begin{array}{l}\text { Symbiotic with } \\
\text { numerous trees }\end{array}$ & $\begin{array}{l}\text { Skin diseases and wound } \\
\text { healing. }\end{array}$ & $\begin{array}{l}\text { Mohamed \& Dix } 1988, \\
\text { Chang \& Miles } 2004 .\end{array}$ \\
\hline 22 & Pleurotus ostreatus (Jacq. ex Fr.) P. & Oyster, abalone & Pleurotaceae & On hardwood trees & Anticancer. & $\begin{array}{l}\text { Yang et al. 2002, } \\
\text { Ajith \& Janardhanan 2007, } \\
\text { Jedinak et al. 2010, De } \\
\text { Silva et al. 2012b. }\end{array}$ \\
\hline 23 & P. sajor-caju (Fr.) Singer. & $\begin{array}{l}\text { Phoenix } \\
\text { mushroom }\end{array}$ & Pleurotaceae & Hardwoods & Lower cholesterol. & $\begin{array}{l}\text { Gunde \& } \\
\text { Cimerman 1995, Chang \& } \\
\text { Miles } 2004 .\end{array}$ \\
\hline 24 & P. sapidus Schulzer \& Kalchbr. & $\begin{array}{l}\text { Oyster or } \\
\text { abalone }\end{array}$ & Pleurotaceae & On hardwood trees & $\begin{array}{l}\text { Skin diseases and wound } \\
\text { healing. }\end{array}$ & $\begin{array}{l}\text { Thimal \& Kluthe } 1998 \text {, } \\
\text { Chang \& Miles } 2004 .\end{array}$ \\
\hline 25 & Russula delica (Pers.) Fr. & $\begin{array}{l}\text { Fragile brittle } \\
\text { gill }\end{array}$ & Russulaceae & Birch & $\begin{array}{l}\text { Skin diseases and wound } \\
\text { healing. }\end{array}$ & Turkoglu et al. 2007. \\
\hline 26 & Scleroderma citrinum Pers. & Earth ball & Sclerodermataceae & Woods & $\begin{array}{l}\text { Skin diseases and wound } \\
\text { healing. }\end{array}$ & $\begin{array}{l}\text { Wakefield \& Elsie } 1964, \\
\text { Chang \& Miles } 2004 .\end{array}$ \\
\hline 27 & Termitomyces heimii Natarajan. & Ejova & Lyophyllaceae & White ant hill & Wound healing & Chandrawati et al. 2014. \\
\hline 28 & T. microcarpus (Berk. \& Broome) R. & Not known & Lyophyllaceae & $\begin{array}{l}\text { Roots of bamboo } \\
\text { stumps }\end{array}$ & Wound healing & Chandrawati et al. 2014. \\
\hline 29 & Trametes versicolor (L.) Lloyd. & Turkey tail & Polyporaceae & Hardwood & $\begin{array}{l}\text { Increase immune system and } \\
\text { depression. }\end{array}$ & $\begin{array}{l}\text { Coles \& Toth 2005, Sun \& } \\
\text { Zhou } 2014 .\end{array}$ \\
\hline 30 & Volvariella speciosa (Fr.) Singer. & $\begin{array}{l}\text { Rose-gilled } \\
\text { grisette }\end{array}$ & Pluteaceae & $\begin{array}{l}\text { Gardens and grassy } \\
\text { fields }\end{array}$ & Antitumor & $\begin{array}{l}\text { Mathew et al. 2008, De } \\
\text { Silva et al. 2012b. }\end{array}$ \\
\hline
\end{tabular}


Table 3 Quantitative of edible and medicinal mushroom species are collected at different altitude from the study area of Walayar valley, Western Ghats of Tamil Nadu, India.

\begin{tabular}{|c|c|c|c|c|}
\hline S. No. & Botanical Name & Available months & Altitude & $\begin{array}{l}\text { Quantitative of } \\
\text { mushroom species } \\
\text { collected per } \\
\text { season/Kg }\end{array}$ \\
\hline 1 & Auricularia auricula (Bull.) J. & July - September & 561 & 159 \\
\hline 2 & Agaricus augustus Fr. & July - September & 568 & 198 \\
\hline 3 & A. bisporus (J.E.Lange) Emil J. & July - September & 550 & 193 \\
\hline 4 & A. campestris $\mathrm{L}$. & June - October & 649 & 189 \\
\hline 5 & A. heterocystis Heinem. & June - October & 523 & 178 \\
\hline 6 & Bovista nigrescens Pers. & August - September & 731 & 166 \\
\hline 7 & Calocybe indica Kuhner ex Donk. & $\begin{array}{l}\text { April- May and July - } \\
\text { August }\end{array}$ & 745 & 158 \\
\hline 8 & Clavulina rugosa (Fr.) Schroet. & July - August & 745 & 169 \\
\hline 9 & Clitocybe nuda (Bull.) H.E. & July - August & 654 & 198 \\
\hline 10 & Coprinus sp. & July - August & 598 & 163 \\
\hline 11 & Daedaleopsis confragosa (Bolton) J. & $\begin{array}{l}\text { April - May and July - } \\
\text { August }\end{array}$ & 823 & 142 \\
\hline 12 & Ganoderma lucidum (Curtis) P. & August - September & 520 & 201 \\
\hline 13 & Hygrocybe sp. (Schaeff.) P. & July - August & 533 & 193 \\
\hline 14 & Lentinus sajor-caju (Fries) Fries. & July - September & 636 & 176 \\
\hline 15 & L. squarrosulus Mont. & July - September & 641 & 188 \\
\hline 16 & L. tuber-regium (Fr.) Fr. & August - September & 685 & 197 \\
\hline 17 & Lycoperdon echinatum Pers. & July - September & 833 & 158 \\
\hline 18 & Marasmius androsaceus (L.) Fr. & August - September & 789 & 172 \\
\hline 19 & Melanoleuca grammopodia Bull. & $\begin{array}{l}\text { March - April } \\
\text { and August - } \\
\text { September }\end{array}$ & 459 & 164 \\
\hline 20 & Mycena galericulata (Scop.) Gray. & $\begin{array}{l}\text { March - April } \\
\text { and August - } \\
\text { September }\end{array}$ & 490 & 139 \\
\hline 21 & Pisolithus arhizus (Scop.) Rauschert. & July - August & 564 & 165 \\
\hline 22 & Pleurotus ostreatus (Jacq. ex Fr.) P. & August - September & 426 & 154 \\
\hline 23 & P. sajor-caju (Fr.) Singer. & $\begin{array}{l}\text { April - May and July - } \\
\text { August }\end{array}$ & 421 & 151 \\
\hline 24 & P. sapidus Schulzer \& Kalchbr. & $\begin{array}{l}\text { April - May and July - } \\
\text { August }\end{array}$ & 464 & 169 \\
\hline 25 & Russula fragilis (Pers.) Fr. & August - September & 693 & 184 \\
\hline 26 & Scleroderma citrinum Pers. & July - September & 712 & 183 \\
\hline 27 & Termitomyces heimii Natarajan. & August - September & 834 & 187 \\
\hline 28 & T. microcarpus (Berk. \& Broome) R. & July - September & 819 & 194 \\
\hline 29 & Trametes versicolor (L.) Lloyd. & July - September & 788 & 181 \\
\hline 30 & Volvariella speciosa $(\mathrm{Fr}$.) Singer. & August - September & 725 & 198 \\
\hline
\end{tabular}

Sarma et al. (2010) reported that some Ethnic Tribes of Western Assam use wild edible mushrooms as food sources. Ethnic tribes such as Garos, Adivashis, Bodos and Rajbangshis of Western Assam consume at least seven species of mushrooms. According to Bhaben et al. (2011), the ethnic tribes of Nagaland, India also use wild edible mushrooms for food. Srivastava et al. (2011) conducted anethnobotanical survey for distribution and utilization of Termitomyces species in Gorakhpur forest division of Uttar Pradesh, India and reported that tribal people and forest dwellers use Termitomyces species as food and for medicinal purposes (not clearly known but used in malnutrition, weakness and their nutritional disorders). Traditional uses and medicinal potential of Cordyceps sinensis has been studied by Panda and Swain (2011) in Sikkim, India and it was found that most local folk healers/traditional healers use Cordyceps in their herbal medicine for the treatment of 21 ailments including cancer, bronchial asthma, bronchitis, TB, diabetes, cough and cold, erectile dysfunction, BHP, jaundice, and alcoholic hepatitis, among others. Pleurotus sajor- 


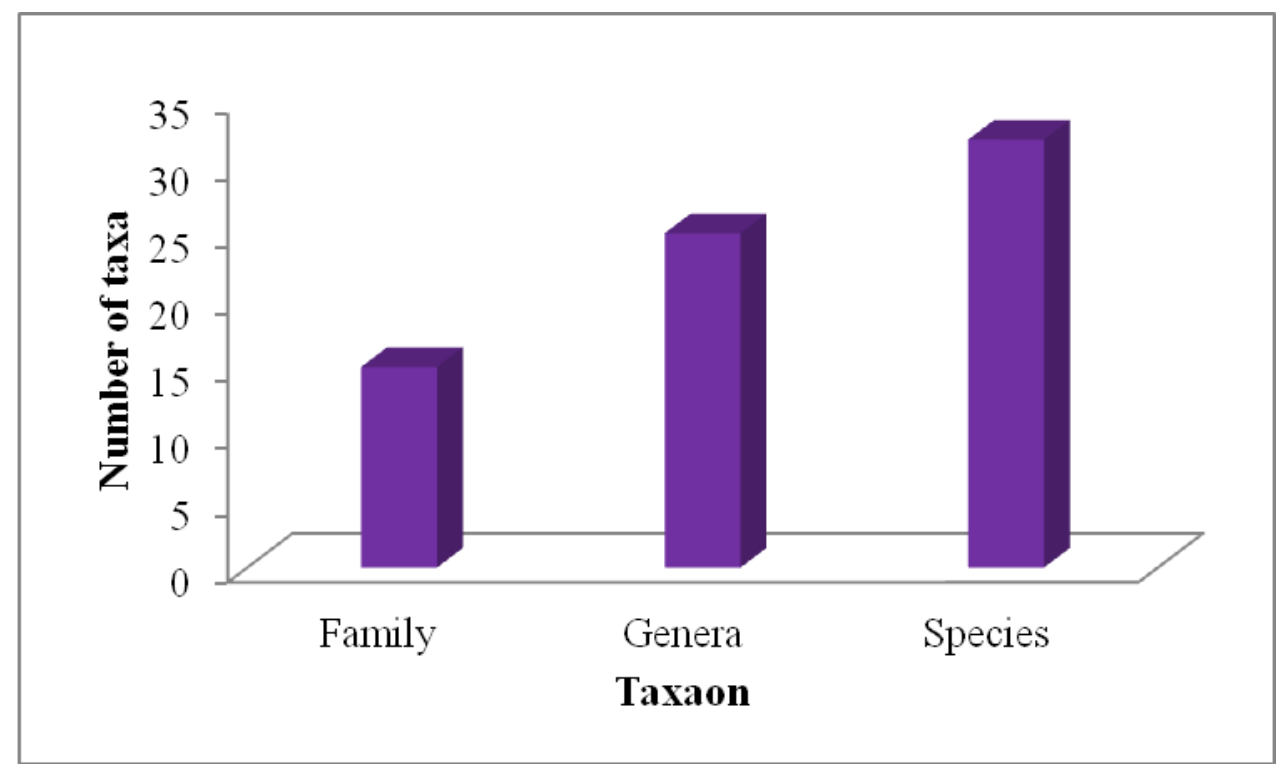

Figs 2 - Taxonomic analysis of medicinal mushroom species in Walayar Valley, Coimbatore district, Tamil Nadu.

caju, Termitomyces heimii, Termitomyces microcarpus, Volvariella volvaceae, Auricularia auriculata, Lentinus fusipes and Lentinus tuberegiumare also consumed by the Kaani tribes of Kanyakumari District in their different recipes (Sargunam et al. 2012). Recently, a survey has been conducted by Sachan et al. (2013) on indigenous knowledge of ethnic tribes from Similipal Biosphere Reserve, Odisha for utilization of wild mushrooms as food and medicine. All these studied medicinal mushrooms are used by several tribals living in the forests for their day-today life food as well as herbal medicinal purposes to cure malnutrition, weakness, and other nutritional disorders such as diarrhoea, high blood pressure, fever, and asthma (Sachan et al. 2013).

As the diversity of edible mushroom species is higher in this valley, it may be considered as potential landscape for wild mushrooms. These mushrooms have been used as ethnomedicines by tribals for treatment of various diseases. Many mushrooms still remain unreported and their nutritional as well as health benefits are unknown to us. If discovered, some of them may have high nutritional value and serve as valuable sources of bioactive compounds with many pharmaceutical applications. Therefore, species-specific cultivation technology should be developed for commercialization and hence conservation.

\section{Acknowledgements}

The authors are thankful to the Irula tribal peoples of Walayar valley, Southern Western Ghats, Coimbatore district, Tamil Nadu. Our sincere thanks to Tamil Nadu State Forest Department is also acknowledged for granting permission to do the work in Walayar Valley.

\section{References}

Ahmad N, Bansal AK, Kidwai JR. 1984a - Effect of PHA-B fraction of Agaricus bisporus lectin on insulin release and $45 \mathrm{Ca}^{2+}$ uptake by islet of Langerhans in vitro. Acta Diabetologica 21 , 63-70.

Ahmad N, Khan MM, Rastogi AK, Kidwai JR.1984b - Effect of age on Agaricus bisporus PHA-B stimulated insulin release and $45 \mathrm{Ca}^{2+}$ uptake in vitro by islets of Langerhans. Acta Diabetologica 21, 349-355.

Ajith TA, Janardhanan KK. 2007 - Indian medicinal mushrooms as a source of antioxidant and antitumor agents. Journal of Clinical Biochemistry and Nutrition 40, 157-162. 
Aletor VA. 1995 - Compositional studies on edible tropical species of mushrooms. Food Chemistry 54, 265-268.

Bandara AR, Rapiord S, Bhat DJ, Kakumyana P, Chamyuang S, Jianchu XU, Kevin DH. 2015 Polyporus umbellatus, an edible-medicinal cultivated mushroom with multiple developed health-care products as food, medicine and cosmetics: a review. Cryptogamie Mycologie 36 (1), 3-42.

Bankhead, Charles. 2000 - Mushrooms may play role in breast cancer prevention and treatment. Web MD Medical News, http://webmd.lycos.com/content/article/1728.53385.

Barros L, Ferreia MJ, Queiros B, Ferreira ICFR, Baptista P. 2007 -Total phenols, ascorbic acid, $\beta$ carotene and lycopene in Portuguese wild edible mushrooms and their antioxidant activities. Food Chemistry 103, 413-419.

Baruah HK, Sing DK, Islam, M. 1971-On the distribution of higher Basidiomycetes in the Sibsagar district, Assam. Bulletin of the Botanical Survey of India 13 (3and4), 285-289.

Benjarong T, Rapior S, Kevin DH, Kathrin W, Stadler M. 2015 - Hericium erinaceus, an amazing medicinal mushroom. Mycological Progress 14, 91.

Bhaben T, Gurung L, Sarma GC. 2011 - Wild edible fungal resources used by ethnic tribes of Nagaland, India. Indian Journal of Traditional Knowledge 10 (3), 512-515.

Bhosle S, Ranadive K, Bapat G, Garad S, Deshpande G, Vaidya J. 2010 - Taxonomy and diversity of Ganoderma from the western parts of Maharashtra (India). Mycosphere 1 (3), 249-262.

Bulakh EM. 2001 - Medicinal mushrooms of the Russian fareast in nature. International Journal of Medicinal Mushrooms 3,125.

Champion SHG, Seth SK. 1968 - A revised survey of the forest types of India. Delhi: Government of India Press.

Chandrawati, Pooja S, Narendra K, Tripathi NN. 2014 - Macrofungal wealth of Kusumhi forest of Gorakhpur, UP, India. American International Journal of Research in Formal, Applied and Natural Sciences 5 (1), 71-75.

Chandulal K, Gopal C, John P. 2013 - Studies on biodiversity of fleshy fungi in Navsari (South Gujarat), India. International Journal of Biodiversity and Conservation 5 (8), 508-514.

Chang R. 1996 - Functional properties of edible mushrooms, Nutrition Reviews 54, 91-93.

Chang ST, Mao XL. 1995 - Hong Kong Mushroom, The Chinese University Press, Hong Kong, pp. 109-110.

Chang ST, Miles PG. 2004 - Mushrooms cultivation, nutritional value, medicinal effect and environmental impact. $2^{\text {nd }}$ Edn., CRC Press, Boca Raton, pp. 450.

Chang YS, Lee SS. 2004 - Utilisation of macrofungi species in Malaysia. Fungal Diversity 15, 1522.

Coles M, Toth B. 2005 - Lack of prevention of large intestinal cancer by VPS, an extract of Coriolus versicolor mushroom. In Vivo 19, 867-871.

Cook FEM. 1995 - Economic Botany Data Collection Standard. Prepared for the International Working Group on Taxonomic Databases for Plant Sciences (TDWG). Kew, Royal Botanic Gardens, pp. 146.

Da Silva MCS, Naozuka J, da Luz JMR, de Assuncao LS, Oliveira PV, Vanetti MCD, Bazzolli DMS, Kasuya MCM. 2012 - Enrichmentof Pleurotus ostreatus mushrooms with selenium in coffee husks. Food Chememistry 131, 558-563.

Dai YC, Yang ZL, Cui BK et al. 2009 - Species diversity and utilization of medicinal mushrooms and fungi in China (review). International Journal of Medicinal Mushrooms 11, 287-302.

Davidson SS, Johnsy G, Selvin Samuel A, Kaviyarasan V. 2012 - Mushrooms in food culture of the Kaani tribe of Kanyakumari district. Indian Journal of Traditional Knowledge 11 (1), $150-153$.

De Silva DD, Rapior S, Fons F, Bahkali AH, Kevin DH. 2012b - Medicinal mushrooms in supportive cancer therapies: an approach to anti-cancer effects and putative mechanisms of action. Fungal Diversity 55, 1-35. 
De Silva DD, Rapior S, Kevin DH, Bahkali, AH. 2012a - Medicinal mushrooms in prevention and control of diabetes mellitus. Fungal Diversity 56, 1-29.

De Silva DD, Rapior S, Sudarman E, Stadler M, Jianchu Xu, Alias SA, Kevin DH. 2013 Bioactive metabolites from macrofungi: ethnopharmacology, biological activities and chemistry. Fungal Diversity 62, 1-40.

Densmore F. 1928 - Uses of plants by the Chippewa Indians. Annual Report of the Bureau of American Ethnology 44, 275-397.

Deshmukh SK. 2004 - Biodiversity of tropical basidiomycetes as sources of novel secondary metabolites. In: Jain PC (ed). Microbiology and Biotechnology for Sustainable Development. CBS Publishers and Distributors, New Delhi, India, pp. 121-140.

Ellis MB. 1971 - Dematiaceous hyphomycetes. The Commonwealth Mycological Institute, England, pp. 1-608.

Enman J, Rova U, Berglund KA. 2007 - Quantification of the bioactive compound eritadenine in selected strains of shiitake mushroom (Lentinus edodes). Journal of Agricultural and Food Chemistry 55, 1177-1180.

Ewart RBL, Kornfeld S, Kipnis DM. 1975 - Effect of lectins on hormone release from isolated rat islets of Langerhans. Diabetes 24, 705-714.

Fasidi IO, Kadiri M. 1990 - Changes in nutritional content of two Nigerian mushrooms $(T$. robustus and L. subnudus) during sporophore development. Die Nahrung 34, 415-420.

Fortes RC, Novaes MRCG, Recova VL, Melo AL. 2009 - Immunological, hematological, and glycemia effects of dietary supplementation with Agaricus sylvaticus on patients'colorectal cancer. Experimental Biology and Medicine 234, 53-62.

Gillman JC. 1957 - Sexual spores, A manual of soil fungi. $2^{\text {nd }}$ Ed, pp 4.

Gogoi Y, Sarma TC. 2012 - An ethnomycological survey in some areas of Dhemaji District (Assam). The Ecoscan 1, 403-407.

Gray AM, Flatt PR. 1998 - Insulin-releasing and insulin-like activity of Agaricus campestris (mushroom). Journal of Endocrinology 157, 259-266.

Guillamon E, Lafuente AG, Lozano M. 2010 - Edible mushrooms: roles in the prevention of cardiovascular diseases. Fitoterapia 81(7), 715-723.

Gunde CN, Cimerman A. 1995 - Pleurotus fruiting bodies contain the inhibitor of 3-Hydroxy-3Methylglutaryl-Coenzyme A reductase-lovastatin. Experimental Mycology 19 (1), 1-6.

Gunde CN. 1999 - Medicinal value of the genus Pleurotus (fr). P Karst (Agaricales s.1. Basidiomycetes). International Journal of Medicinal Mushrooms 1, 69-80.

Harsh NSK, Rai BK, Ayachi SS. 1993 - Forest fungi and tribal economy-a case study in Baiga tribe of Madhya Pradesh (India). Journal of Forestry 9, 270-279.

Hilden K, Makela MR, Lankinen P, Lundell T. 2013 - Agaricus bisporus and related Agaricus species on lignocellulose production of manganese peroxidase and multicopper oxidases. Fungal Genetics and Biology55, 32-41.

Hyde KD, Bahkali AH, Moslem MA. 2010 - Fungi - an unusual source for cosmetics. Fungal Diversity, 43, 1-9.

Jagadish LK, Krishnan VV, Shenbhagaraman R, Kaviyarasan V. 2009 - Comparative study on the antioxidant, anticancer and antimicrobial property of Agaricus bisporus (J. E. Lange) Imbach before and after boiling. African Journal of Biotechnology 8, 654-661.

Jedinak A, Dughgaonkar S, Jian J, Sandusky G, Silva D. 2010 - Pleurotus ostreatus inhibits colitisrelated colon carcinogenesis in mice. International Journal of Medicinal Mushrooms26, 643-650.

Jing Xu, Xiaobo Yang, Qiang Lin. 2014 - Chemistry and biology of Pestalotiopsis-derived natural products. Fungal Diversity 66, 37-68.

Johnsy G, Davidson Sargunam S, Dinesh MG, Kaviyarasan V. 2011 - Nutritive value of edible wild mushrooms collected from the Western Ghats of Kanyakumari district. Botany Research International 4(4), 69-74. 
Kabita D, Albinus L, Dibyendu P, Kumar LJ. 2014 - Ethnomycological knowledge on wild edible mushroom of Khasi tribes of Meghalaya, North-Eastern India. European Academic Research 2 (3), 3433-3443.

Kala CP. 2009 - Chemical composition and nutritional value of European species of wild growing mushrooms: A review. Food Chemistry 113, 9-16.

Kasuga A, Aoyagi Y, Sugahara T. 1995 - Antioxidant activity of fungus Suillus bovines. Journal of Food Science60, 1113-1115.

Khaund P, Joshi SR. 2013 - Wild edible macrofungal species consumed by the Kashi tribe of Meghalaya, India. Indian Journal of Natural Products and Resources 4 (2), 197-204.

Kinsalin VA, Kumar PS, Duraipandiyan V, Ignacimuthu S, Al-Dhabi NA. 2014 - Antimicrobial activity of methanol extracts of some traditional medicinal plants from Tamil Nadu, India. Asian Journal of Pharmaceutical and Clinical Research 7(1), 36-40.

Komoda Y, Shimizu M, Sonoda Y, Sato Y. 1989 - Ganoderic acid and its derivatives as cholesterol synthesis inhibitors. Chemical and Pharmaceutical Bulletin 37, 531-533.

Lin WY, Yang MJ, Hung LT, Lin LC. 2013 - Antioxidant properties of methanol extract of a new commercial gelatinous mushrooms (white variety of Auricularia fuscosuccinea) of Taiwan. African Journal of Botechnology 12 (43), 6210-6221.

Lin ZB, Zhang HN. 2004 - Antitumor and immunoregulatory activities of Ganoderma lucidum and its possible mechanisms. Acta Pharmacologica Sinica 25, 1387-1395.

Lindequist U, Niedermeyer THJ, Julich WD. 2005 - The pharmacological potential of mushrooms -Review.Evidence-Based Complementary and Alternative Medicine2 (3), 285-299.

Liu JQ, Wang CF, Peng XR, Qiu MH. 2011 - New alkaloids from the fruiting bodies of Ganoderma sinense. Natural Products and Bioprospecting 1, 93-96.

Liu X, 2014 - A food blood nourishing tea granule for improving nutritional anemia. China patent application CN103493937A.

Luangharn T, Hyde KD, Chukeatirote E. 2014 - Proximate analysis and mineral content of Laetiporus sulphureus strain MFLUCC 12-0546 from northern Thailand. Chiang Mai Journal of Science 41, 765-770.

Luo DQ, Chen YP, Zhang J, Shi BZ, Yang ZQ, Chen C. 2013 - A new glycine derivative and a new indole alkaloid from the fermentation broth of the plant endophytic fungus Pestalotiopsis podocarpi isolated from the Chinese Podocarpaceae plant Podocarpus macrophyllus. Helvetica Chimica Acta 96, 309-312.

Mathew J, Sudheesh PN, Rony KA, Smina TP, Janardhanan KK. 2008 - Antioxidant and antitumor activities of cultured mycelium of culinary-medicinal paddy straw mushroom Volvariella volvacea (Bull.: Fr.) Singer (Agaricomycetideae). International Journal of Medicinal Mushrooms 10 (2), 139-147.

Mattila P, Suonpaa K, Piironen V. 2000 - Functional properties of edible mushrooms, Nutrition 16 (7-8), 694-696.

Mattila PK, Konko M, Eurola J, Pihlava J, Astola L, Vahteristo V, Hietaniemi J, Kumpulainen N, Valtonen V, Piironen V. 2000 - Contents of vitamins, mineral elements and some phenolic compounds in the cultivated mushrooms. Journal of Agricultural and Food Chemistry 49, 2343-2348.

Mitchell TG, Perfect JR. 1995 - Cryptococcosis in the era of AIDS-100 years after the discovery of Cryptococcus neoformans. Journal of Clinical Microbiology Reviews 8, 515-548.

Mohamed SH, Dix NJ. 1988 - Resource utilization and distribution of Coprinus comatus, Coprinus atramentarius, Lacrimaria velutina and Melanoleuca grammopodia. Transactions of the British Mycological Society 90, 255-263.

Mohanan S. 2014 - Macro fungal diversity in the Western Ghats, Kerala, India: members of Russulaceae. Journal of Threatened Taxa 6 (4), 5636-5648.

Moradali MF, Mostafavi H, Ghods S, Hedjaroude GA. 2007 - Immunomodulating and anticancer agents in the realm of macromycetes fungi (macrofungi). International Immunopharmacology 7, 701-724. 
Mortimer PE, Karunarathna SC, Li QH, Gui H, Yang XQ, Yang XF, He J, Ye L, Guo JY, Li HL, Sysouphanthong P, Zhou DQ, Xu JC, Hyde KD. 2012 - Prized edible Asian mushrooms: ecology, conservation and sustainability. Fungal Diversity 56 (1), 31-47.

Ohtsuka S, Ueno S, Yoshikumi C, Hirose F, Ohmura Y, Wada T, Fujii T, Takahashi E. 1997 Polysaccharides having an anticarcinogenic effect and a method of producing them from species of Basidiomycetes. UK Patent No. 1331513.

Oyetayo VO. 2009 - Free radical scavenging and antimicrobial properties of extracts of wild mushrooms. Brazilian Journal of Microbiology 40 (2), 380-386.

Panda AK, Swain KC. 2011 - Traditional uses and medicinal potential of Cordyceps sinensis of Sikkim. Journal of Ayurveda and Integrative Medicine 2 (1), 9-13.

Poongkode GK, Sakthi S. 2001 - Dietary efficacy of the edible mushroom Agaricus bisporus (J. Lge) imbach in modulating the risk of coronary heart disease in rats. Perspectives of Medicinal Mushrooms in Healthcare and Nutrition in the $21^{\text {st }}$ Century. 12-14 Septemeber Kiev, Ukraine. Abstract in International Journal of Medicinal Mushrooms 3(2-3),pp. 200.

Pushpa, Purushothama. 2012 - Biodiversity of mushrooms in and around Bangalore (Karnataka), India. American-Eurasian Journal of Agricultural \& Environmental Sciences 12 (6), 750759.

Qin DW, Gu Z, Guo JU. 2015 - Medicinal mushroom for prevention of disease of modern civilization. Evidence Based Complementary and Alternative Medicine doi:10.1155/2015/812725.

Rai M, Tidke G, Wasser SP. 2005 - Therapeutic potential of mushrooms. Natural Products Radiance 4 (4), 246-257.

Rajesh KA, Tapwal S, Pandey RK, Borah D, Borgohain J. 2013 - Macro-fungal diversity and nutrient content of some edible mushrooms of Nagaland, India. Bioscience 5 (1), 1-7.

Richter C, Wittstein K, Kirk PM, Stadler M. 2015 - An assessment of thetaxonomy and chemotaxonomy of Ganoderma. Fungal Diversity 71, 1-15.

Rios JL, Andujar I, Recio MC, Giner RM. 2012 - Lanostanoids from fungi: A group of potential anticancer compounds. Journal of Natural Products 75, 2016-2044.

Sachan SKS, Patra JK, Thatoi HN. 2013 - Indigenous knowledge of ethnic tribes for utilization of wild mushrooms as food and medicine in Similipal biosphere reserve, Odisha, India. Journal of Agricultural Technology 9 (2), 335-348.

Samorini G. 2001- Fungi Hallucinogeni. Studi etnomicologici. Telesterion. Dozza, Bologna, Italy, pp. 250.

Sargunam SD, Johnsy G, Dinesh MG, Kaviyarasan V. 2012 - Nutritive value of edible wild mushrooms collected from the Western Ghats of Kanyakumari district. Botany Research International 4 (4), 69-74.

Sarma TC, Sarma I, Patiri BN. 2010 - Wild edible mushrooms used by some ethnic tribes of western Assam. The Bioscan 3, 613-625.

Semwal KC, Stephenson SL, Bhatt VK, Bhatt RP. 2014 - Edible mushrooms of the Northwestern Himalaya, India: a study of indigenous knowledge, distribution and diversity. Mycosphere 5 (3), 440-461.

Sharma SK, Atri NS. 2014 - Nutraceutical composition of wild species of genus Lentinus Fr. From Nothern India. Current Research in Environmental \& Applied Mycology 4 (1), 11-32.

Sing NI, Sing SM, Th C. 2002 - Fleshy fungi of Manipur. In: Vij SP, Kondo K, Sharma ML, Gupta A (eds). Plant Genetic Diversity: Exploaration, Evaluation, Conservation. Afficiated East West Press Pvt. Ltd., New Delhi, India, pp. 9-13.

Sing NI, Sing SM. 1993 - Edible fleshy fungal flora of Manipur. Biofuels4 (2), 153-158.

Srivastava B, Dwivedi AK, Pandey VN. 2011 - Ethnobotanical survey, distribution and utilization of Termitomyces species in Gorakhpur forest division. Plant Science Feed1 (3), 28-33.

Stadler M, Hoffmeister D. 2015 - Fungal natural products-the mushroom perspective. Frontiers in Microbiology 127, 1-4. 
Sun S. 2014 - One kind of traditional Chinese medicine composition for treating diabetes. China patent application CN103550660A.

Sun Y, Zhou X. 2014 - Purification, initial characterization and immune activities of polysaccharides from the fungus, Polyporus umbellatus. Food Science and Human Wellness 3, 73-78.

Tapwal A, Kumar R, Pandey S. 2013 - Diversity and frequency of macro fungi associated with wet ever green tropical forest in Assam, India. Biodiversitas 14 (2), 73-78.

Thatoi H, Singdevsachan SK. 2014 - Diversity, nutritional composition and medicinal potential of Indian mushrooms: A review. African Journal of Biotechnology 13, 523-545.

Thawthong A, Karunarathna SC, Thongklang N, Chukeatirote E, Kakumyan P, Chamyuang S, Rizal M, Mortimer, PE, Xu, JC, Callac P, Hyde KD. 2014 - Discovering and Domesticating Wild Tropical Cultivatable Mushrooms. Chiang Mai Journal of Science 41, 731-764.

Thimal R, Kluthe R. 1998 - The nutritional database for edible mushrooms. Ernahrung 22, 63-65.

Turkoglu A, Duru ME, Mercan N, Kivrak I, Gezer K. 2007 - Antioxidant and antimicrobial activities of Laetiporous sulphureus (Bull.) Murrill. Food Chemistry 101 (1), 267-273.

Turkoglu A, Duru ME, Mercan N. 2007 - Antioxidant and Antimicrobial Activity of Russula delica Fr: An Edidle Wild Mushroom. Eurasian Journal of Analytical Chemistry 2 (1), 5467.

Villares A, Vivaracho LM, Guillamon E. 2012 - Structural features and healthy properties of polysaccharides occurring in mushrooms. Agriculture 2 (4), 452-471.

Wakefield, Elsie M. 1964 - The Observer's Book of Common Fungi (Observer's Pocket Series No. 19) (3rd printing ed.). Frederic Warne \& Co Ltd. OCLC 748994120.

Wang G, Zhao J, Liu J, Huang Y, Zhong JJ, Tang W. 2007 - Enhancement of IL-2 and IFNgamma expression and NK cells activity involved in the anti-tumor effect of ganoderic acid Me in vivo. International Immunopharmacology 7, 864-70.

Wasser SP, Weis AL. 1999a - Medicinal properties of substances occurring in higher Basidiomycetes mushrooms: current perspectives (review). International Journal of Medicinal Mushrooms 1 (1), 31-62.

Wasser SP. 2002 - Medicinal mushrooms as a source of antitumor and immunomodulating polysaccharides. Applied Microbiology and Biotechnology 60, 258-74.

Yang JH, Lin HC, Mau JL. 2002 - Antioxidant properties of several commercial mushrooms. Food Chemistry 77, 229-235.

Yu S, Weaver V, Martin K, Cantorna MT. 2009 - The effects of whole mushrooms during inflammation. BMC Immunology 10, 12.

Yuan Z, He P, Cui J. Takeuchi H. 1998 - Hypoglycemic effect of water-soluble polysaccharide from Auricularia auricula-judae Quel. on genetically diabetic KK-Ay mice. Bioscience, Biotechnology, and Biochemistry 62, 1898-1903.

Ziegenbein FC, Hanssen HP, Konig WA. 2006 - Secondary metabolites from Ganoderma lucidum and Spongiporus leucomallellus. Phytochemistry 67, 202-211. 